\title{
THE COMPACT RANGE PROPERTY AND $C_{0}$
}

\author{
by NEIL E. GRETSKY and JOSEPH M. OSTROY
}

(Received 6 June, 1985)

The purpose of this short note is to make an observation about Dunford-Pettis operators from $L^{1}[0,1]$ to $C_{0}$. Recall that an operator $T: E \rightarrow F$ (where $E$ and $F$ are Banach spaces) is called Dunford-Pettis if $T$ takes weakly convergent sequences of $E$ into norm convergent sequences of $F$. A Banach space $F$ has the Compact Range Property (CRP) if every operator $T: L^{1}[0,1] \rightarrow F$ is Dunford-Pettis. Talagrand shows in his book [2] that $C_{0}$ does not have the CRP. It is of interest (especially in mathematical economics [3]) to note that every positive operator from $L^{1}[0,1]$ to $C_{0}$ is Dunford-Pettis.

For a Banach lattice $Y$, call a Schauder basis order-compatible if the natural projections are positive operators. Certainly in this case, the basis elements themselves are positive.

THEOREM 1. Let $Y$ be a Banach lattice with order compatible Schauder basis. Then every positive linear operator $T: L^{1}[0,1] \rightarrow Y$ is a Dunford-Pettis operator.

Proof. By a theorem of Bourgain given in [1], it suffices to show that $T \circ i$ : $L^{\infty}[0,1] \rightarrow Y$ is a compact operator, where $i: L^{\infty}[0,1] \rightarrow L^{1}[0,1]$ is the natural injection. To fix notation, let $y_{1}, y_{2}, y_{3}, \ldots$ be the basis of $Y$ and let $P_{n}$ and $Q_{n}$ be the associated projections defined by

and

$$
P_{n}\left(\sum_{i=1}^{\infty} a_{i} x_{i}\right)=\sum_{i=1}^{n} a_{i} x_{i}
$$

$$
Q_{n}=I-P_{n} \quad(n=1,2,3, \ldots)
$$

By hypothesis, each of these projection operators is positive. Now let $f$ be in the unit ball of $L^{\infty}[0,1]$. We compute

so that

$$
\begin{aligned}
(T \circ i) f & =\left(P_{n} \circ T \circ i\right) f+\left(Q_{n} \circ T \circ i\right) f \\
& =T_{n} f+R_{n} f
\end{aligned}
$$

$$
\begin{aligned}
\left\|(T \circ i) f-T_{n} f\right\| & =\left\|R_{n} f\right\| \\
& \leq\left\|R_{n} \chi_{[0,1]}\right\|,
\end{aligned}
$$

since $|f| \leq \chi_{[0,1]}$ and $Y$ is a Banach lattice. Given $\varepsilon>0$, there is $N$ such that $\left\|R_{n} \chi_{[0,1]}\right\|<\varepsilon$ for $n \geq N$. So for any $f$ in the unit ball of $L^{\infty}$, and $n \geq N$, we have

$$
\left\|(T \circ i) f-T_{n} f\right\|<\varepsilon .
$$

Consequently, $\left\|(T \circ i)-T_{n}\right\|<\varepsilon$. Since each $T_{n}$ has finite dimensional range, $T \circ i$ is a compact operator. Thus $T$ is Dunford-Pettis.

A consequence of this theorem is that every regular operator from $L^{1}[0,1]$ to $Y$ is Dunford-Pettis. (Recall that an operator between two Banach lattices $E$ and $F$ is called

Glasgow Math. J. 28 (1986) 113-114. 
regular if it is the difference of two positive operators.) In general, the regular operators are a proper subset of the bounded linear operators; in two special cases, however, it is well known that equality holds: one case is when $F$ is an order complete $C(K)$ with $K$ compact; the other is when $E$ is $L^{1}(\mu)$ and $F$ is the range of a positive, continuous projection in its bidual.

Examples of Banach lattices which fit into the framework of the theorem are $C_{0}$ and $l_{1}$. In the case of $l_{1}$, every operator from $L^{1}[0,1]$ to $l_{1}$ is regular (second case above) and thus is Dunford-Pettis. (Of course, it is already obvious that this must be so because $l_{1}$ has the RNP and hence the CRP.) The space $C_{0}$ does not fit either of the two cases and, in fact as mentioned above, $C_{0}$ does not have the CRP. One final note: $L^{1}[0,1]$ itself fits the second case and every operator from $L^{1}[0,1] \rightarrow L^{1}[0,1]$ is regular but not necessarily Dunford-Pettis, as the identity operator shows.

COROLlaRY 2. $L^{1}[0,1]$ cannot have an order compatible Schauder basis.

\section{REFERENCES}

1. J. Bourgain, Dunford-Pettis operator on $L^{1}$ and the Radon-Nikodyn property, Israel $J$. Math. 37 (1980), 34-27.

2. M. Talagrand, The Pettis integral, Mem. Amer. Math. Soc. No. 307, (Rhode Island, 1984).

3. N. E. Gretsky and J. M. Ostroy, Thick and thin market non-atomic exchange economies, in Advances in Equilibrium Theory, Lecture Notes in Economics and Mathematical Systems No. 244 (1985), 107-130.

Department of Mathematics

UNIVERSITY OF CALIFORNIA

RIVERSIDE

CAlifornia 92521
DEPARTMENT OF ECONOMICS

UNIVERSITY OF CALIFORNIA

LOS ANGELES

CALIFORNIA 90024 